\title{
Study of the Calculation Method for Direction Angle of Equivalent Coefficient of V-belt Friction
}

\author{
Zhaoli Lin \\ Chongqing College of Electronic Engineering, Chongqing 400054, China
}

\begin{abstract}
Keywords: circular deformation, radial deformation, direction angle $\varphi$, the equivalent coefficient of
\end{abstract} friction.

\begin{abstract}
According to the actual transmit situation of v-belt , analyze radial deformation and circular deformation of any position of dynamicarcarea of driving wheel and driven wheel and the method and Formula Equivalent Coefficient of friction direction angle is given; on the basis of a practical example, a relationship figure of $\alpha$ and $\varphi$ has been obtained.In regard to direction angle of friction , find the regular of the equivalent coefficient of friction of driving wheel and driven wheel in driven wheel the equivalent coefficient of friction decrease with the increase of direction angle; in driving wheel the equivalent coefficient of friction increase with the increase of absolute of direction angle; According to the equivalent coefficient of friction of the driving wheel no more than $\frac{\mu}{\sin \frac{\theta}{2}}$, and the formula of limit skid torque ,then emplains the numble of actual limit skid torque more the number of theory limit skid torque.
\end{abstract}

\section{Preface}

In $\mathrm{V}$ belt transmission, Angle is divided into static arc area and dynamic arc area. In the static arc area there is only the radial sliding, in the dynamic arc area, there is a circumferential sliding and radial sliding, so for the dynamic arc area of arbitrary cross section $\mathrm{V}$ belt on the friction force direction is not purely along the circumferential or radial, but along the contact surface (main direction) in a certain direction. For circumferential and radial sliding friction should be the friction in the circumferential and radial direction projection. The main direction and circumferential tangential direction angle is friction direction Angle(hereinafter referred to as direction Angle) ${ }^{[1]}$.In $\mathrm{V}$ belt transmission process, the equivalent friction coefficient is not a fixed value, but a variable related to belt transmission process, Because the direction Angle is introduced, the formula of equivalent friction coefficient is more accurate.

Known the direction angle of any position in dynamic arc area, we can determine the friction, then get the ultimate torque of actual needs used to avoid slipping. And we can also explain the situation that the actual ultimate slipping torque is greater than the theoretical ultimate slipping torque. But at home and in the world, there are a few studies at direction angle and did not give a reasonable direction angle formula, in some literature ${ }^{[2,3]}$ refers to the direction angle in the driving wheels, but part of the derivation process is controversial conclusion, in addition the conclusion also have deviation with the actual situation. This paper analyzes the driving wheel and the driven wheel circumferential deformation and radial deformation, gives the formula used to calculation direction angle of the driving wheel and the driven wheel, and using software to draw the relationship diagram between direction angle and dynamic arc angle. Because in the static arc area there is only the radial sliding, so the direction angle is 90 degrees. This paper mainly discusses the direction angle in dynamic arc area.

\section{The Calculation Of Driven Wheel Direction Angle}

This paper studies circumferential deformation and radial deformation of any position in dynamic arc area from the causes of direction Angle being, then we can calculate that the angle at arbitrary location deformation, it is equal and opposite compare with direction angle. Thus we can calculate the direction angle of any position in dynamic arc area. 


\subsection{Circumferential Deformation}

Circumferential deformation is the tight tension is greater than the loose side tension cause relative deformation. This article is using sliding rate formula to calculate circumferential deformation.

Based on slip rate formula of reference documentation ${ }^{[4]}{ }^{150}$, slip rate of any position deformation in dynamic arc area is:

$$
\varepsilon_{\mathrm{hi}}=\frac{\mathrm{v}_{\mathrm{i}}-\mathrm{v}_{2}}{\mathrm{v}_{2}}
$$

Among them: $v_{i}$ is the speed of any point in dynamic arc area.

In the driven wheel dynamic arc area, time $d_{t}$ turned the arc length $d_{\alpha}$, as shown in figure 1 :

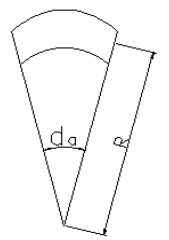

From figure 1 we known:

Fig 1 micro arc segment of driven wheel

Among them: $\mathrm{R}$ is the radius of belt wheel

$$
\operatorname{Rd}_{\alpha}=\mathrm{v}_{2} \mathrm{~d}_{\mathrm{t}}
$$

According to the relative sliding of the $\mathrm{V}$ belt and driven belt wheel, tangential distortion in the case of micro segment $i$ as follows:

Simultaneous (1), (2), (3) :

$$
\Delta \mathrm{l}_{\mathrm{x}}=\left(\mathrm{v}_{\mathrm{i}}-\mathrm{v}_{2}\right) \mathrm{d}_{\mathrm{t}}
$$

$$
\Delta \mathrm{l}_{\mathrm{x}}=\varepsilon_{\mathrm{hi}} \operatorname{Rd}_{\alpha}
$$

Based on slip ratio formula of reference documentation ${ }^{[5]}{ }^{49-51}$, the formula between slip rate and the tension of any position deformation in dynamic arc area is:

$$
\varepsilon_{\mathrm{hi}}=\frac{\mathrm{F}_{\mathrm{i}}-\mathrm{F}_{2}}{\mathrm{sE}}
$$

Among them: $\mathrm{s}$ is the original cross-sectional area.

Simultaneous(4),(5), we know the formula of circumferential deformation at driven wheel is:

\subsection{Radial Deformation:}

$$
\Delta \mathrm{l}_{\mathrm{x}}=\frac{\mathrm{F}_{\mathrm{i}}-\mathrm{F}_{2}}{\mathrm{sE}} \mathrm{Rd}_{\alpha}
$$

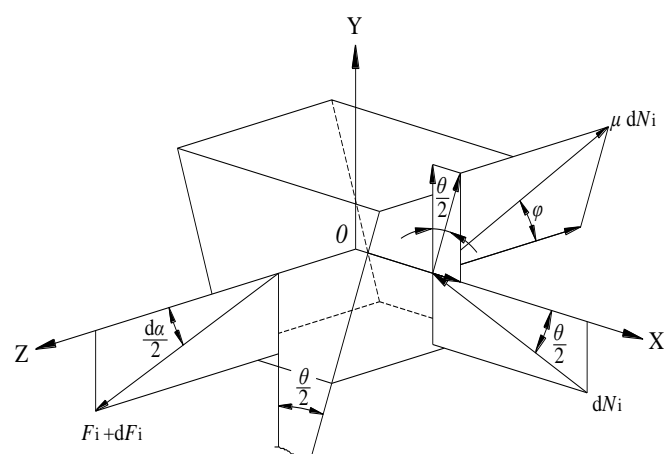

Fig $2 \mathrm{~V}$ belt force diagram in the micro-arc $\mathrm{i}$

In $\mathrm{V}$ belt in the process of actual transmission, due to the dynamic arc area of different location of the axial force is different, so as to make the different radial deformation.

According to figure 2 do stress analysis :

$$
\begin{gathered}
\mathrm{d}_{\mathrm{N}_{\mathrm{i}}} \cos \frac{\theta}{2}-\mu \mathrm{d}_{\mathrm{N}_{\mathrm{i}}} \sin \varphi \sin \frac{\theta}{2}=\mathrm{F}_{\mathrm{h}_{\mathrm{i}}} \\
\mathrm{d}_{\mathrm{F}_{\mathrm{i}}}=2 \mu \mathrm{d}_{\mathrm{N}_{\mathrm{i}}} \cos \varphi \\
2 \mathrm{~d}_{\mathrm{N}_{\mathrm{i}}} \sin \frac{\theta}{2}+2 \mu \mathrm{d}_{\mathrm{N}_{\mathrm{i}}} \sin \varphi \cos \frac{\theta}{2}=\mathrm{F}_{\mathrm{i}} \mathrm{d}_{\alpha}
\end{gathered}
$$

Among them: $\mathrm{F}_{\mathrm{h}_{\mathrm{i}}}$ is axial force of paragraph $\mathrm{i}$

arc, the direction along ative $\mathrm{x}$ axis.

Similarly, i-1 segmental arc force analysis as follows: 


$$
\begin{gathered}
\mathrm{d}_{\mathrm{N}_{\mathrm{i}-1}} \cos \frac{\theta}{2}-\mu \mathrm{d}_{\mathrm{N}_{\mathrm{i}-1}} \sin \varphi \sin \frac{\theta}{2}=\mathrm{F}_{\mathrm{h}_{\mathrm{i}-1}} \\
2 \mathrm{~d}_{\mathrm{N}_{\mathrm{i}-1}} \sin \frac{\theta}{2}+2 \mu \mathrm{d}_{\mathrm{N}_{\mathrm{i}-1}} \sin \varphi \cos \frac{\theta}{2}=\mathrm{F}_{\mathrm{i}-1} \mathrm{~d}_{\alpha}
\end{gathered}
$$

Cross section of $\mathrm{V}$ belt as shown in figure 3:

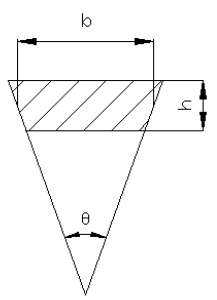

Among them:

Fig 3 Cross section of belt

b: the width of the neutral layer of belt

$\mathrm{h}$ : the thickness of belt

According to the elastic deformation formula of reference documentation ${ }^{[6]}$,we known the axial deformation of segment $i$ is :

Among them:

$$
\Delta \mathrm{l}_{\mathrm{i}}=\frac{\mathrm{F}_{\mathrm{h}_{\mathrm{i}} \mathrm{b}}}{\mathrm{EA}}
$$

$\mathrm{A}=\operatorname{Rd}_{\alpha} \mathrm{h}$

Simultaneous (7), (12) :

$$
\Delta \mathrm{l}_{\mathrm{i}}=\frac{\left(\cos \frac{\theta}{2}-\mu \sin \varphi \sin \frac{\theta}{2}\right) \mathrm{d}_{\mathrm{N}_{\mathrm{i}}} \mathrm{b}}{\mathrm{EA}}
$$

Similarly, axial deformation of segment $\mathrm{i}-1$ is:

$$
\Delta \mathrm{l}_{\mathrm{i}-1}=\frac{\left(\cos \frac{\theta}{2}-\mu \sin \varphi \sin \frac{\theta}{2}\right) \mathrm{d}_{\mathrm{N}_{\mathrm{i}-1} \mathrm{~b}}}{\mathrm{EA}}
$$

through(13)and(14), we can get the axial deformation increment of segment $\mathrm{i}$ is:

$$
\Delta \mathrm{l}_{\mathrm{h}_{\mathrm{i}}}=\frac{\left(\cos \frac{\theta}{2}-\mu \sin \varphi \sin \frac{\theta}{2}\right)\left(\mathrm{d}_{\mathrm{N}_{\mathrm{i}}-} \mathrm{d}_{\mathrm{N}_{\mathrm{i}-1}}\right) \mathrm{b}}{\mathrm{EA}}
$$

According to (9), (11), (15) :

$$
\Delta \mathrm{l}_{\mathrm{h}_{\mathrm{i}}}=\frac{\left(\cos \frac{\theta}{2}-\mu \sin \varphi \sin \frac{\theta}{2}\right) \frac{\mathrm{d}_{\alpha} \mathrm{b}}{2 \sin \frac{\theta}{2}+2 \mu \sin \varphi \cos \frac{\theta}{2}}\left(\mathrm{~F}_{\mathrm{i}}-\mathrm{F}_{\mathrm{i}-1}\right)}{\mathrm{EA}}
$$

$\frac{1}{2} \frac{\left(\cos \frac{\theta}{2}-\mu \sin \varphi \sin \frac{\theta}{2}\right) \mathrm{b}}{\mathrm{EA}\left(\sin \frac{\theta}{2}+\mu \sin \varphi \cos \frac{\theta}{2}\right)}=\mathrm{k}$

Through the conversion:

$$
\Delta \mathrm{l}_{\mathrm{h}_{\mathrm{i}}}=\mathrm{kd}_{\alpha} \mathrm{d}_{\mathrm{F}_{\mathrm{i}}}
$$

By the axial deformation into radial deformation as shown in figure 4:

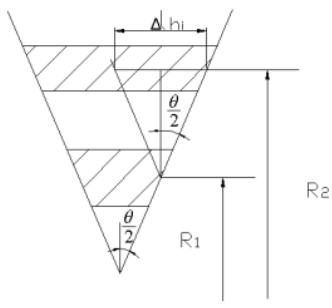

Fig 4 deformation diagram after conversion

By the figure we known:

$$
\Delta \mathrm{R}=-\frac{\frac{1}{2} \mathrm{kd}_{\alpha} \mathrm{d}_{\mathrm{F}_{\mathrm{i}}}}{\tan \frac{\theta}{2}}
$$

\subsection{The Calculation of $\varphi$ :}

In the process of actual transmission, $\varphi$ is positive or negative, thus it is rules that the value of direction angle that point to the wheel center is negative, the opposite direction is positive.

As shown in figure 5: 


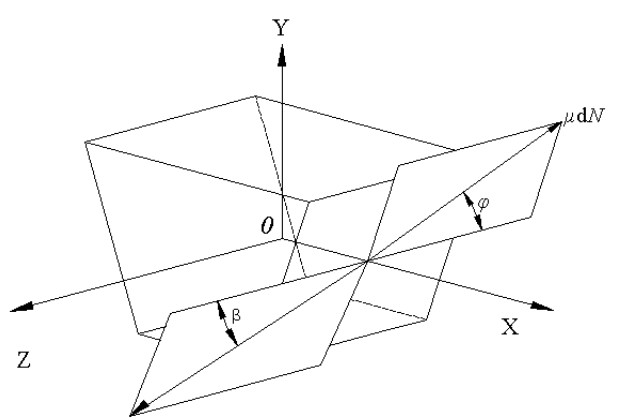

Fig 5 deformation angle diagram of $\mathrm{V}$ belt

$$
\cos \beta=-\frac{\Delta \mathrm{l}_{\mathrm{x}}}{\Delta \mathrm{R} / \cos \frac{\theta}{2}}
$$

Among them: $\beta$ is intersection angle between the circumferential deformation and radial deformation, it is equal to $\varphi$ and opposite with it.

Simultaneous (6), (18), (19):

$$
\cot \varphi=\frac{2 \mathrm{R} \sin \frac{\theta}{2}\left(\mathrm{~F}_{\mathrm{i}}-\mathrm{F}_{2}\right)}{\operatorname{sEKd}_{\mathrm{F}_{\mathrm{i}}}}
$$
$[7,8]$.

According to the equivalent friction coefficient formula of references documentation ${ }^{[1]}$ 42, [5] 16-17,

$$
\mu_{\mathrm{v}}=\frac{\mu \cos \varphi}{\sin \frac{\theta}{2}+\mu \sin \varphi \cos \frac{\theta}{2}}
$$

Simultaneous k, (8), (9), (20), (21), we known friction direction Angle formula is:

$$
\varphi=\sin ^{-1} \frac{\mu_{\mathrm{v}}^{2}}{\mu\left[\frac{4 \mathrm{R}^{2} \mathrm{~h}}{\mathrm{bs}}\left(1-\mathrm{e}^{-\mu_{\mathrm{v}} \alpha}\right)+\mu_{\mathrm{v}}^{2}\right] \tan \frac{\theta}{2}}
$$

By the formula (22) we known, the size of friction force direction Angle $\varphi$ is relationship with the $\mathrm{V}$ belt type (main parameters is the groove angle $\theta$ ), and the friction coefficient of flat belt.

Assumes that the friction force coefficient of belt is $\mu=0.4$, pulley diameter $\mathrm{R}=60 \mathrm{~mm}$, select $\mathrm{v}$ belt model is $\mathrm{z}$, the parameters in the following table 1 .

Tab $1 \mathrm{~V}$ belt model is $\mathrm{z}$ parameter table

\begin{tabular}{|c|c|c|c|c|}
\hline name & $\mathrm{b}$ & $\mathrm{H}$ & $\theta$ & $\mathrm{s}$ \\
\hline figure & 8.5 & 6.0 & $40^{\circ}$ & 51 \\
\hline
\end{tabular}

Iterate using MATLAB software according to the above parameters, in the process of iteration, the errors is decreases, when the precision of the absolute error less than $0.1^{\circ}$, stop the iteration. When flat belt friction coefficient $\mu=0.4$, the relation diagram between $\varphi$ and $\alpha$ as shown in figure 6 .

The figure 6 shows that in the process of the $\mathrm{V}$ belt transmission, on the belt of driven wheel, $\varphi$ is positive, value range is $\left(0^{\circ}, 90^{\circ}\right)$, and the value is gradually become smaller as the dynamic arc angle increase, until close to $0^{\circ}$. Using the same method, we can get relation diagram between different friction coefficient of flat belt and different friction direction angle of groove

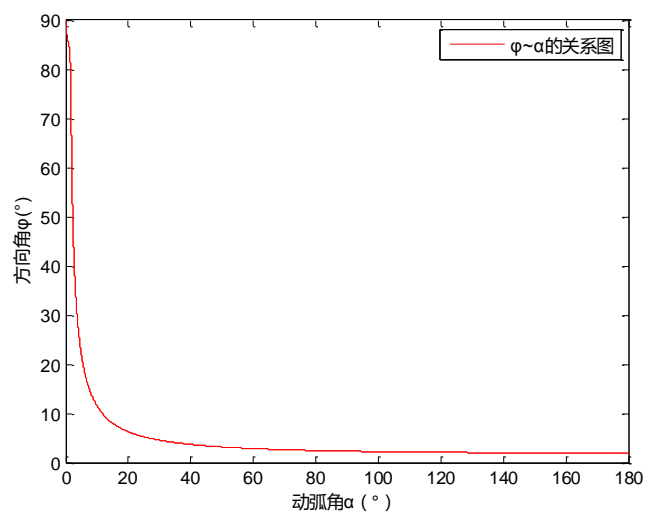

Fig 6 The relation diagram between $\varphi$ and $\alpha$ in driven wheel 
angle and dynamic arc angle, according to the diagram, the friction force direction angle in the dynamic arc area not only value range is constant and the same change trend.

\section{The Calculation of Driving Wheel Direction Angle:}

The calculation process of driving wheel direction angle and the calculation process of driven wheel direction angle is similar, the calculation process is omitted, only part of the calculation formula is given as follows.

\subsection{Circumferential Deformation:}

\subsection{Radial Deformation:}

$$
\Delta \mathrm{l}_{\mathrm{x}}=\frac{\mathrm{F}_{1}-\mathrm{F}_{\mathrm{i}}}{\mathrm{sE}} \mathrm{Rd}_{\alpha}
$$

By the axial deformation into radial deformation (as shown in figure 7), the radial deformation formula is:

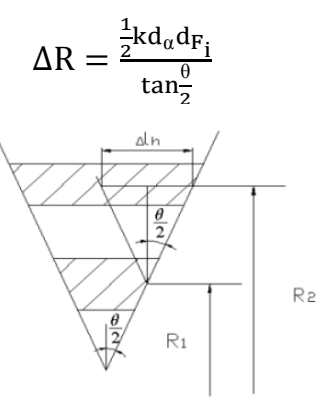

\subsection{The Calculation of $\varphi$ :}

Fig 7 Radial deformation figure

$$
\varphi=-\sin ^{-1} \frac{\mu_{\mathrm{v}}{ }^{2}}{\mu\left[\frac { 4 \mathrm { R } ^ { 2 } \mathrm { h } } { \mathrm { bs } } \left(\mathrm{e}^{\left.\left.\mu_{\mathrm{v}}{ }^{\alpha}-1\right)+\mu_{\mathrm{v}}^{2}\right] \tan \frac{\theta}{2}}\right.\right.}
$$

Because the equivalent friction coefficient is positive, Thus $\varphi$ has constraints: $\mu_{\mathrm{v}} \geq 0$.

Iterate using MATLAB software according to the above parameters, in the process of iteration, the errors is decreases, when the precision of the absolute error achieve $0.1^{\circ}$ can be considered to be more precise diagram between $\varphi$ and $\alpha$ (figure 8 ).

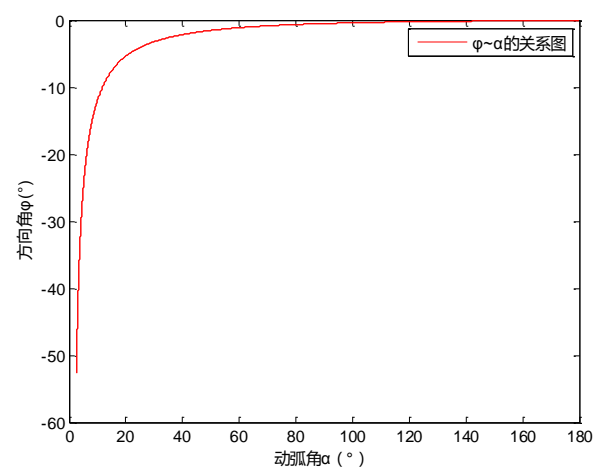

Fig 8 the relation diagram between $\varphi$ and $\alpha$ in driving wheel

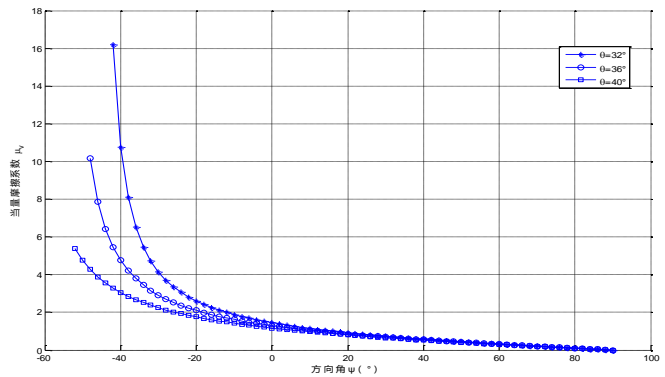

Fig $9_{\varphi \sim \mu_{\mathrm{v}}}$ Relation graph 
The figure 8 shows that in the process of the $\mathrm{V}$ belt transmission, in the belt of driving wheel, the value of $\varphi$ is negative or zero, the scope is $\left(-52^{\circ} 0^{\circ}\right.$ ] and the value become bigger and bigger as dynamic arc angle increase, until to $0^{\circ}$. Using the same method, we can get relation diagram between different friction coefficient of flat belt and different friction direction angle of groove angle and dynamic arc angle, according to the diagram, the friction force direction angle in the dynamic arc area have the same change trend but the range has changed (remains negative or zero).

\section{Effect of Direction Angle $\varphi$}

to equivalent friction coefficient

From formula (21)we known, equivalent friction coefficient $\mu_{\mathrm{v}}$

of the $\mathrm{V}$ belt is relate to groove angle $\theta$, friction coefficient $\mu$ of flat belt and friction force direction angle $\varphi$. friction force direction angle has Positive and negative. When $\varphi=0^{[9]}$,formula (21) translate into:

$$
\mu_{\mathrm{v}}=\frac{\mu}{\sin \frac{\theta}{2}}
$$

Friction force direction angle of driven wheel is positive, when $\varphi>0$,formula (21) as follows:

$$
\mu_{\mathrm{v}}=\frac{\mu \cos \varphi}{\sin \frac{\theta}{2}+\left|\mu \sin \varphi \cos \frac{\theta}{2}\right|}
$$

Friction force direction angle of driving wheel is not positive, when $\varphi \leq 0$,

formula (21) as follows:

$$
\mu_{\mathrm{v}}=\frac{\mu \cos \varphi}{\sin \frac{\theta}{2}-\left|\mu \sin \varphi \cos \frac{\theta}{2}\right|}
$$

For example, when $\mu=0.4, \theta=32^{\circ}, 36^{\circ}, 40^{\circ}$, the relation diagram as follows (as shown in fig 9)

From fig 9 we known when the groove angle is changed, in the driven wheel dynamic arc area, equivalent friction coefficient decreases with the increase of friction direction angle and the equivalent friction coefficient $\mu_{\mathrm{v}}<\frac{\mu}{\sin \frac{\theta}{2}}$. In the driving wheel dynamic arc area, equivalent friction coefficient increases

with the increase of absolute value friction direction angle and the equivalent friction coefficient $\mu_{\mathrm{v}} \geq \frac{\mu}{\sin \frac{\theta}{2}}$.

\section{The Direction Angle of the Influence of V Belt Skid Torque Limit}

Belt creep usually occur in the first working radius small belt wheel, usually occurs on the driving wheel. Is about to skid when the critical effective pull [4], 158.

Among them: represents the driving wheel Angle

$$
F_{e c}=2\left(F_{0}\right)_{\min } \frac{1-\frac{1}{e^{f \alpha}}}{1+\frac{1}{e^{f \alpha}}}
$$

Critical for $\mathrm{V}$ belt slippage theory effective pull type as follows:

$$
F_{e c}=2\left(F_{0}\right)_{\min } \frac{e^{\mu_{v} \alpha}-1}{e^{\mu_{v} \alpha}+1}
$$

Determine the effective tensile load torque, the largest load torque which is the ultimate slip torque is:

$$
\begin{gathered}
T_{\max }=F_{e c} \times R_{2} \\
=2\left(F_{0}\right)_{\min }\left(1-\frac{2}{e^{\mu_{v} \alpha+1}}\right) \times R_{2}
\end{gathered}
$$

Generally think the direction Angle of $0^{\circ}$, then on the type of; According to as described in this paper, the direction Angle is variable in a driving wheel dynamic arc area, makes, so type (29) in the larger, which shows the actual limit slip torque is greater than the theoretical limit slip torque.

\section{Conclusion}

(1), based on the radial deformation and circumferential deformation of V belt 
Analysis of the driven wheel and driving wheel dynamic formula of arc zone of direction Angle, and combining the parameters of the simulation diagram.

(2), in the process of transmission, from the pulley $\mathrm{V}$ belt in equivalent friction coefficient decreases with the increase of friction direction Angle, and satisfaction; And driving wheel equivalent friction coefficient decreases with the increase of friction direction Angle, and satisfaction;

(3), according to the driving wheel equivalent friction coefficient, combined with the skid torque limit formula, can explain the limit of actual slippage phenomenon of the slippage of the torque is greater than the theoretical limit torque.

\section{References}

[1]. Xiang-Qiu. Lin qing-Liao. Ming-Xie. Formula of equivalent friction coefficient of V belt transmission correction. "Journal of chongqing institute of technology (natural science edition)" (4) $21.2007: 42$

[2]. Lin-Zhu. Yi xiang-Liu. Calculate the equivalent friction coefficient of $\mathrm{V}$ belt transmission impact Angle research. Journal of Harbin commercial university (natural science edition), 22 (1). 2006.2

[3]. Da yu-Zheng. Yi xiang-Liu Shen-Chen. A new method for calculating v-belt equivalent friction coefficient. The Chinese mechanical engineering (13) 15. 2004.7

[4]. Liang Gui-Pu. Mechanical design. The eighth edition. Higher education press. 2006.5:150,158

[5]. Xiang-Qiu. Study on the design theory of belt type continuously variable transmission [D]. Chongqing: Chongqing University of Technology, 2009:16-18.58

[6]. Hong wen-Liu. Mechanics of materials. Fourth ed. higher education press.2004.1:33-34

[7]. Bei qi-Sun. [D]. Of CVT transmission mechanism. Chongqing: Chongqing University of Technology, 2007:21-23

[8]. Oliver L R, Henderson D D. Torque Sensing Variable Speed VBelt Drive.\#Paper 720708 presented at SAE Farm[C] (Construction and Industrial Machinery Meeting. Mliwaukee: [s. n.].1972:21-29

[9]. Lin-Zhu. Da yu-Zheng. Study on the calculation of equivalent friction coefficient of V. Mechanical design and research, Vol. third, No. twenty-fourth,.2008.11:67-68 